\title{
Numerical Simulation of Shrinkage Formation Behavior with Consideration of Solidification Progress during Mold Filling Using Stabilized Particle Method*
}

\author{
Naoya Hirata and Koichi Anzai \\ Department of Metallurgy, Graduate School of Engineering, Tohoku University, Sendai 980-8579, Japan
}

Solidification begins during pouring in casting processes, and the pouring rate influences the solidification pattern and soundness of products. In this research, the combined simulation of fluid flow and solidification simulation was performed based on the MPS method (a particle method) to analyze the behavior of shrinkage formation from the beginning of pouring to the end of solidification, and the influence of pouring rate on the solidification pattern and shrinkage shape of pure Al casting was investigated. Solidification simulation and stabilized flow simulation programs based on the particle method were combined considering the temperature-dependency of density. The proposed method was applied to shrinkage formation analysis based on the influence of pouring velocity on the shrinkage formation behavior. The results showed that the depth and shape of shrinkage agreed well with corresponding experimental results. The shrinkage formation behavior could also be directly calculated. [doi:10.2320/matertrans.F-M2017811]

(Received January 19, 2017; Accepted March 1, 2017; Published May 25, 2017)

Keywords: shrinkage formation behavior, flow simulation, solidification simulation, coupled simulation, particle method, MPS method

\section{Introduction}

A shrinkage defect formation is strongly influenced by solidification patterns. Therefore, casting design such as risers, chills and tapers of the products thickness are well discussed in the design phase, so as to avoid closed loops of solidification patterns in the products. Recently, numerical simulation to predict shrinkage formation have been put into practical use by considering the shrinkage flow behavior directly ${ }^{1-7)}$. However, most of conventional shrinkage prediction algorithms is applied to the solidification simulations separately from flow simulations. Therefore, there is a few report which mentions an influence of solidification during the pouring, whereas in the actual casting process, a solidification is considered to begin during the pouring, and the pouring speed influences the solidification patterns, and consequent soundness of the products ${ }^{8,9}$. These facts show the importance of considering the influence of solidification during the pouring; a shape of the unsolidified molten pool and its influence on the thermal convection patterns are not negligible to predict shrinkage formation precisely.

Recently, particle methods, which are based on a fully Lagrangian method, have been developing to calculate complex phenomena occurring in the casting processes ${ }^{10-12)}$. Nowadays, the typical particle methods for computational continuum dynamics are usually categorized into two methods, SPH (Smoothed Particle Hydrodynamics) ${ }^{11)}$ method and MPS (Moving Particle Semi-implicit) ${ }^{12)}$ method. Discrete objects are used as calculation elements in the particle methods, and they can move freely in the space. This feature allows the particle methods to simulate the heat and mass transfer phenomena that are observed in the casting process more easily and directly than other methods that use the calculation lattice.

A series of phenomena from the pouring to the solidification shrinkage formation in the conical mold casting has been

*This Paper was Originally Published in Japanese in J. JFS 88 (2016) 11 17. simulated by Ichimiya et al. using the SPH method ${ }^{13,14)}$. Kazama et al. also have tried to calculate shrinkage formation behavior by using the SPH method ${ }^{15)}$. An effect of feeding behavior on the shrinkage formation in a low pressure die casting process has been calculated by Cleary et al. by using the SPH method ${ }^{16)}$. Hirata et al. have reported shrinkage formation behavior calculation by using the MPS method ${ }^{17-19,21)}$.

The algorithm of the MPS method is simple and similar to that of the conventional finite difference method (FDM). Also, a spatial distribution of particles (calculation elements) are uniform ${ }^{20}$, which gives an advantage in the stability of calculation including phase change. However, a calculation stability in the flow calculation remains unknown for the integrated simulation from the pouring to the solidification ${ }^{20-22)}$. Therefore, stabilized flow simulation based on the MPS method was proposed in this study. And the fluid flow and solidification simulations were coupled by using the MPS method. Then, the integrated simulation was performed from the pouring to the end of solidification to directly reproduce the influence of the pouring speed on the solidification patterns and the shape of shrinkage.

\section{Numerical Method}

The flow and solidification calculation program were used in this study, which the authors have been developing based on the MPS method. A summary of the calculation algorithms is shown in this section.

\subsection{Heat transfer and solidification simulation}

Heat transfer equations are described as follows.

$$
\begin{gathered}
\frac{D H}{D t}=\lambda \nabla^{2} T \\
\Delta Q=\frac{\Delta t}{R}\left(T_{1}-T_{2}\right)
\end{gathered}
$$

Here, $H$ represents the enthalpy per unit volume $\left(\mathrm{J} \cdot \mathrm{m}^{-3}\right)$; $t$, time $(\mathrm{s}) ; \lambda$, thermal conductivity $\left(\mathrm{W} \cdot \mathrm{m}^{-1} \cdot \mathrm{K}^{-1}\right) ; T$, tem- 
perature $(\mathrm{K}) . \Delta Q\left(\mathrm{~J} \cdot \mathrm{m}^{-2}\right)$ is the thermal flux per unit area in time $\Delta t(\mathrm{~s}) . R$ is heat resistance $\left(\mathrm{m}^{2} \cdot \mathrm{K} \cdot \mathrm{W}^{-1}\right)$, and $T_{1}(\mathrm{~K})$ and $T_{2}(\mathrm{~K})$ are the surface temperatures of materials 1 and 2 at the interface between the materials. The left-hand side of the eq. (1), $D / D t$, is the Lagrangian derivative, having the same significance as temporal differentiation.

Heat transfer equations (eqs. (1) and (2)) were transformed into the particle interaction operations using the Laplacian model of the MPS method, and the effect of solidification was considered by using the enthalpy method ${ }^{23)}$.

\subsection{Flow simulation}

The governing equations for incompressible flow consist of the continuity equation and the Navier-Stokes equation as follows.

$$
\frac{D \rho}{D t}=0, \quad \frac{D \boldsymbol{u}}{D t}=-\frac{1}{\rho} \nabla p+v \nabla^{2} \boldsymbol{u}+\boldsymbol{f}
$$

Here, $\rho$ indicates density $\left(\mathrm{kg} \cdot \mathrm{m}^{-3}\right) ; \boldsymbol{u}$, velocity vector $\left(\mathrm{m} \cdot \mathrm{s}^{-1}\right) ; p$, pressure $(\mathrm{Pa}) ; v$, kinematic viscosity $\left(\mathrm{m}^{2} \cdot \mathrm{s}^{-1}\right)$; and $f\left(\mathrm{~m} \cdot \mathrm{s}^{-2}\right)$, the body force vector including gravity. The left-hand side of the second equation, $D / D t$, is the Lagrangian derivative, having the same significance as temporal differentiation. Flow calculation by the MPS method is based on the predictor-corrector method similar to that in the case of the FDM or other Eulerian methods ${ }^{20)}$.

The improved multiple relaxation method proposed in the previous report was found to be effective to improve the flow calculation accuracy and stability ${ }^{22)}$. Therefore, the method was adopted in this study. The flowchart for the flow simulation with the improved multiple relaxation method is shown in the Fig. 1. The Poisson equation is solved more than once in the multiple relaxation method. Accordingly the velocity and position are also corrected more than once during one time step. The improved method uses the adjustment coefficient $c_{p o i}$ in the source term in the Poisson equation for the pressure. The calculation conditions are expressed as the combination of the number of multiple relaxations and $c_{p o i}$. For example, in the case of two times of multiple relaxations and $c_{p o i}=0.5$, then the condition is expressed as MR2-0.5.

\subsection{Temperature dependence of density}

Temperature-dependent particle size was introduced to describe a volume change with solidification. The specific size of the particle $i, r_{0, i}$, is described using following equation.

$$
r_{0, i}=\left(\frac{M_{i}}{\rho_{i}}\right)^{1 / d}
$$

Here, $M_{i}$ and $\rho_{i}$ are mass $(\mathrm{kg})$ and density $\left(\mathrm{kg} \cdot \mathrm{m}^{-3}\right)$ of the particle $i$, respectively. The $d$ is the number of space dimensions. Assuming that the masses of each particle are constant, we can calculate the solidification shrinkage using the temperature-dependent density as described in eq. (4) ${ }^{19)}$.

\section{Influence of Pouring Speed on Shrinkage Formation Behavior}

Two different pouring speed were examined for the same casting design to analyze the influence of solidification pattern during the pouring on the shrinkage formation behavior.

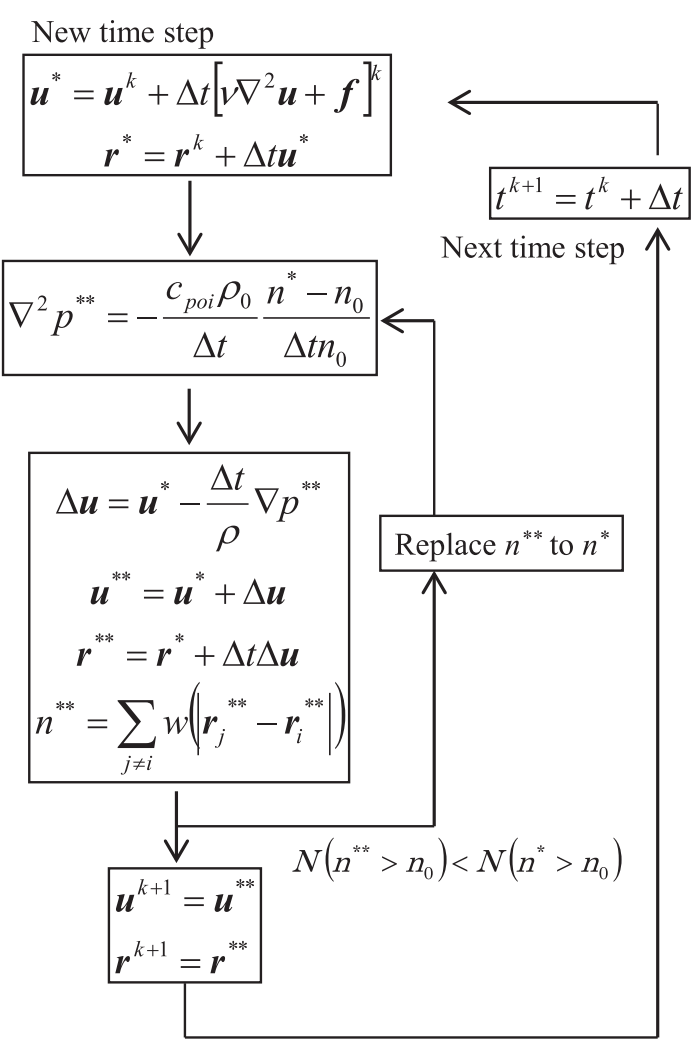

Fig. 1 Flow chart for the flow simulation using modified multiple relaxation method ${ }^{22)}$.

The flow simulation was coupled with the heat transfer and solidification simulation with consideration of temperature-dependent density. The effect of viscosity and inertia force were taken into account until the pouring is finished, and only the gravitational force was considered to improve the calculation stability after filled up or the pouring finished, as described in the previous study ${ }^{19,21)}$.

\subsection{Calculation model}

Figure 2 shows the casting design. Physical properties used in the calculation are shown in Table $1^{21)}$. Initial particle size was $2 \mathrm{~mm}$ uniformly. Thus, we use 108767 particles for the mold, 19535 particles for the casting. Inflow gate with $196 \mathrm{~mm}^{2}$ area (49 particles) was set at the upper part of the casting. The amount of the melt was $390 \mathrm{~g}$, and the temperature was $948 \mathrm{~K}$. The melt was poured from the gate by $0.2 \mathrm{~m} \cdot \mathrm{s}^{-1}$ or $0.057 \mathrm{~m} \cdot \mathrm{s}^{-1}$, then the pouring time was $4 \mathrm{~s}$ or $14 \mathrm{~s}$, respectively. The viscosity was assumed constant above the melting point. The effect of solidification was taken into account by fixing the positions of the particles in space at which a temperature was below the melting point. The heat transfer coefficient between the casting and the mold was assumed to be $2000 \mathrm{~W} \cdot \mathrm{K}^{-1} \cdot \mathrm{m}^{-2}$ so as to match the solidification time to the experimental result. A constant condition of the improved multiple relaxation method was used through the calculation in this study, and the value was MR5-0.5 which showed the most stable calculation in the previous study $^{22)}$. The conditions of MR2-0.5 and MR1-1.0 were also examined to compare the calculation accuracy and stability. Normal flow simulation was applied for $0.5 \mathrm{~s}$ after the pouring finished to wait until the flow becomes slow. After $0.5 \mathrm{~s}$ 
from the pouring finished, only the gravitational force was considered, and the calculation was continued to the end of solidification.

FDM calculation was also conducted to compare the results of the particle method and the conventional method. ADSTEFAN Ver.2014 was used for FDM calculation. Calculation lattice size was $1 \mathrm{~mm}$. Flow and heat transfer calculation was conducted with consideration of latent heat release. In a similar way to the particle method, flow calculation was continued until $0.5 \mathrm{~s}$ after the pouring finished. The obtained temperature field was transferred to the solidification simulation to predict a shrinkage shape. The velocity of the elements was assumed to be zero if the temperature of the elements is under the melting point. A simple feeding model was used to predict the shrinkage shape. In the feeding model, the molten metal is fed from the upper part of a liquid region including the elements showing the solidification shrinkage.

\subsection{Results and discussions}

\subsubsection{Velocity field}

The effect of the improved multiple relaxation method on the pressure stability for slow fluid flow calculation was reported in the previous study ${ }^{22)}$. The multiple relaxation method was also effective in the case of complex shaped casting with the shoulder. Here, the velocity field with relatively

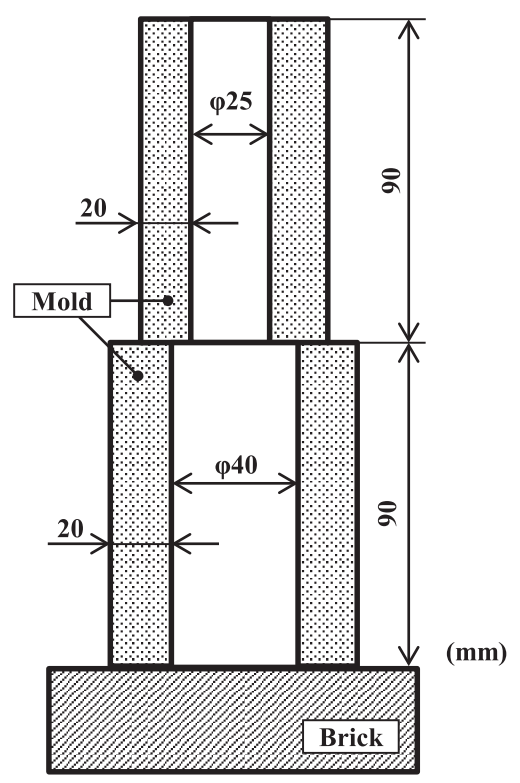

Fig. 2 Mold design ${ }^{21)}$.

Table 1 Physical properties for simulation.

\begin{tabular}{lcc}
\hline & Casting & Mold \\
\hline Materials & Pure Al & Cast Iron \\
\hline Density, $\rho / \mathrm{kg} \cdot \mathrm{m}^{-3}$ & $\begin{array}{c}2500 \text { (Liquid) } \\
2700 \text { (Solid) }\end{array}$ & 7500 \\
\hline Thermal conductivity, $\lambda / \mathrm{W} \cdot \mathrm{m}^{-1} \cdot \mathrm{K}^{-1}$ & 200 & 33 \\
\hline Specific heat, $c_{p} / \mathrm{J} \cdot \mathrm{kg}^{-1} \cdot \mathrm{K}^{-1}$ & 1100 & 550 \\
\hline Kinematic Viscosity, $\nu / \mathrm{m}^{2} \cdot \mathrm{s}^{-1}$ & $10^{-5}$ & - \\
\hline Melting temperature, $T_{m} / \mathrm{K}$ & 933 & - \\
\hline Latent heat, $H_{f} / \mathrm{kJ} \cdot \mathrm{kg}^{-1}$ & 389 & - \\
\hline
\end{tabular}

higher velocity was shown to observe the effect of the multiple relaxation method on the velocity decay. Only flow calculation was conducted, and the effect of solidification was ignored in this section 3.2.1. Figure 3 shows the calculation results under various multiple relaxation conditions. Figure 3(a) and (b) show the case of MR1-1.0, Fig. 3(c) and (d) are the case of MR2-0.5, and Fig. 3(e) and (f) are the results of MR5-0.5, Fig. 3(g) is the result of FDM, respectively. The figures show the cross section with the thickness equal to two particles. The color in the results of particle methods indicates vertical velocity component, and the absolute value of the velocity was shown for the results of FDM because the software has no function to output the velocity components. The results without the multiple relaxation method (Fig. 3(a) and (b)) show significant velocity decay, and almost no flow behavior was observed at the bottom part of the casting. After the level of the casting rises over the shoulder, the flow in the lower part almost stopped. As the number of multiple relaxations increase, downward flow remains clearly after the level rise over the shoulder. These results show that the improved multiple relaxation method improves calculation accuracy not only for the slow fluid flow calculation but relatively faster fluid flow calculation. This is because the improved multiple relaxation method reduces an excess movement and consequent oscillation behavior of particles, as shown in the previous study ${ }^{22)}$.

The flow calculation by using the MPS method shows lower accuracy compared with the upwind scheme, and the velocity decay become obvious with the existence of a free surface $^{20)}$. The result by FDM is shown in the Fig. 3(g) to compare with the results calculated by the particle method. Figure 3(e) and (f) show that the modified multiple relaxation method has given remarkable improvement to the velocity decay, however the decay remains compared with the results of FDM. Therefore, further improvement is required for the flow calculation accuracy.

A non-continuous flow as shown in the Fig. 3(a) to (f) is caused by ignoring the effect of surface tension and contraction flow. Clustering occurs in actual cases, therefore the effect of surface tension and a negative pressure should be considered to calculate inflow behavior more precisely. However, such effects will have almost no effect on the solidification behavior during the pouring in the case of simple model as shown in this study. Therefore we neglected the effect of surface tension and negative pressure in this study. In addition, the use of the improved multiple relaxation method also has almost no effect on the shrinkage formation behavior shown in the next section. However, the method will work effectively in the case of more complex shaped casting in which the flow and solidification interact each other and have some effect on the shrinkage formation.

\subsubsection{Shrinkage formation behavior}

Two kinds of solidification sequences with different pouring speed are shown in Figs. 4 and 5. The flow and solidification were calculated using particle method with the improved multiple relaxation method. Only the case of MR5-0.5 is shown in this study. The figures show the cross section with the thickness equal to two particles, and the color indicates the solid fraction. Figure 4 is the result when the pouring duration is $4 \mathrm{~s}$. The solid fraction increases because some 


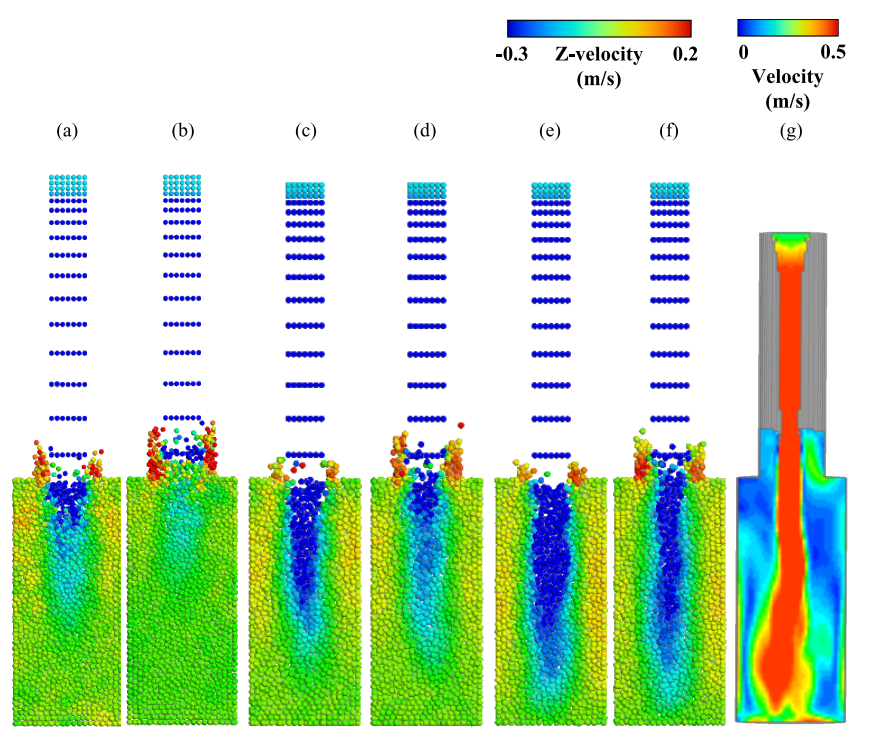

Fig. 3 Velocity distribution. (a) to (f) : vertical velocity distribution calculated by the particle method. (g) : velocity distribution calculated by FDM. (a) MR1-1.0 $t=3.1 \mathrm{~s}$, (b) MR1-1.0 $t=3.2 \mathrm{~s}$, (c) MR2-0.5 $t=3.1 \mathrm{~s}$ (d) MR2-0.5 $t=3.2 \mathrm{~s}$, (e) MR5-0.5 $t=3.1 \mathrm{~s}$, (f) MR5-0.5 $t=3.2 \mathrm{~s}$, (g) $\mathrm{FDM} t=3.2 \mathrm{~s}$.

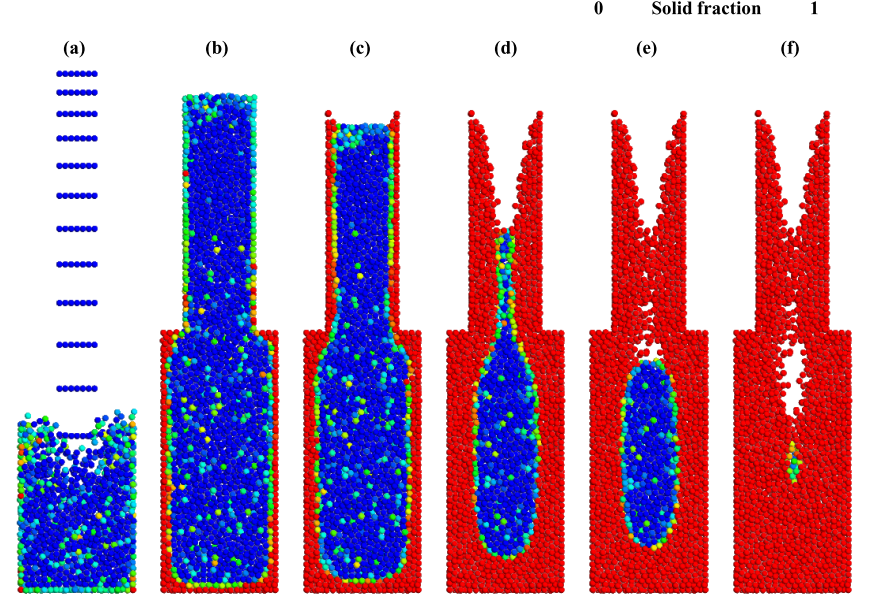

Fig. 4 Calculated shrinkage behavior : $t_{\text {pour }}=4 \mathrm{~s}$. (a) $2 \mathrm{~s}$, (b) $4.2 \mathrm{~s}$, (c) $6 \mathrm{~s}$, (d) $10 \mathrm{~s}$, (e) $11 \mathrm{~s}$, (f) $14.7 \mathrm{~s}$.

amount of latent heat was absorbed during the pouring. However no solidification shell generated owing to its high pouring rate. A thin shell grew at the bottom of the casting after the pouring finished. After that, the solidification shell grew equally from all of the mold walls, resulting in a large and deep shrinkage. Figure 5 is the result when the pouring duration is $14 \mathrm{~s}$. The solid fraction increases immediately after the pouring starts. More than half part of the casting had already solidified when the pouring finished. A solidification shrinkage occurred simultaneously with the pouring. Therefore the height of the casting is shorter than the that of rapid pouring.

\subsubsection{Shrinkage shape}

Figures 6 and 7 show the cross section of the castings under different pouring speeds. Figures 6 and 7 show the results of $4 \mathrm{~s}$ and $14 \mathrm{~s}$, respectively. In each figure, (a) shows the experimental result, (b) is the particle number density distribu-

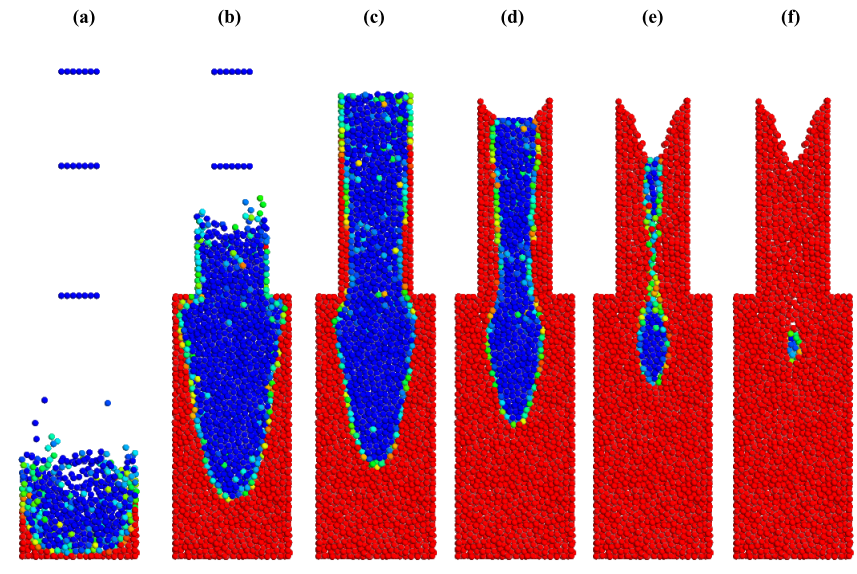

Fig. 5 Calculated shrinkage behavior : $t_{\text {pour }}=14 \mathrm{~s}$. (a) $4 \mathrm{~s}$, (b) $12 \mathrm{~s}$, (c) $14.5 \mathrm{~s}$, (d) $17 \mathrm{~s}$, (e) $19 \mathrm{~s}$, (f) $20 \mathrm{~s}$. (a)

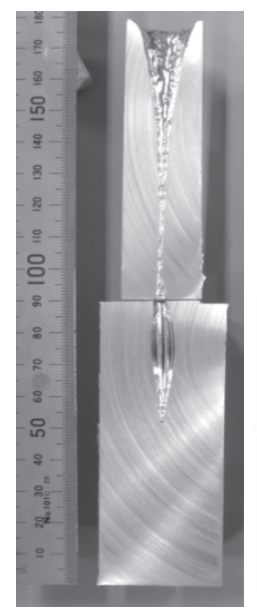

0.95 Particle number 1.05 density, $n_{i} /-$

(c)

(d)

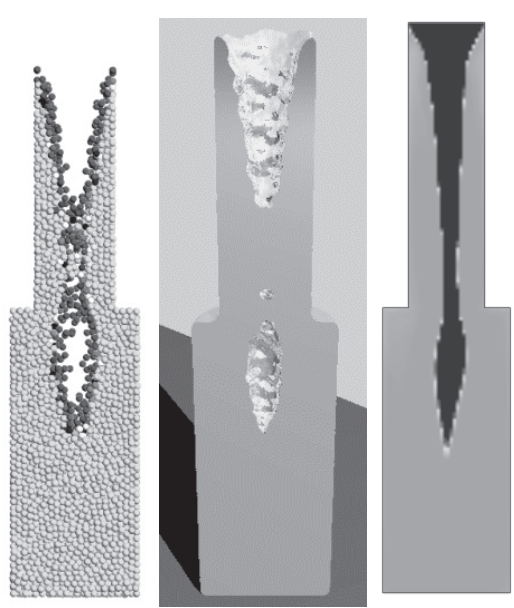

Fig. 6 Experimental and calculated results of shrinkage shape: $t_{\text {pour }}=4 \mathrm{~s}$. (a) Experimental result, (b) Particle number density, (c) Visualized by POV-RAY, (d) FDM.

tion calculated by using the particle method, showing the cross section with a thickness equal to two particles. The particles with low $n_{i}$ are determined as the surface particles, and indicated with black color; the threshold was $n_{i}<0.985$ in this study ${ }^{22)}$. (c) is a visualized result of particle method calculation by using the ray tracing software POV-Ray Ver.3.7 and the blob method. The upper part is enlarged because of the limitation of the positioning of the lighting and the camera. (d) is the result of FDM by using ADSTEFAN.

Figure 6(a) shows that the rapid pouring resulted in thin and long shrinkage reaching the deep part of the casting, whereas Fig. 7(a) shows that the slow pouring makes small shrinkage porosity in the middle of the casting. Comparing Fig. 6(b), (c) and Fig. 7(b), (c), the particle method results agreed well with the experimental results. The results by 
FDM differed slightly to the experimental results especially in the case of the slow pouring. Because the solidification shrinkage during the pouring was ignored in this calculation. The latest version of the commercial software is introduced the effect of solidification shrinkage during the pouring, and it enables to reproduce the influence of the pouring speed on the height of castings. However, the special program is required to treat the shrinkage behavior simultaneously with the flow calculation; further coupling simulation will be hard by using FDM or by the other conventional method.

\subsubsection{Shape of casting}

The height of the casting in the case of rapid pouring is longer than that of slow pouring, as shown in Figs. 6(a) and 7(a). The results of particle method calculation reproduced the difference well. However, the calculated heights of castings were shorter than the experimental results. The difference occurs by ignoring adhesion behavior and the heat resistance between the casting and the mold. These phenomena would cause the lowering duration of the upper surface to be longer than expected. Therefore, the prediction accuracy of the shrinkage shape will increase by considering the adhesion effect and the use of smaller particle size.

The surfaces of objects visualized by the blob method are iso-surfaces of some scalar fields ${ }^{24)}$. The definition is similar to describe the objects in the particle method. Therefore the blob method is suitable for the visualization of particle method results. However, the detailed shape of the edge of the casting or the thickness of the shrinkage differs from the experimental results. This is because that the weight functions used in the particle method and the blob method were different. In addition, some fittings of parameters are required for the blob method. The same parameters were used in this study for the visualization of different pouring speed results in this study. However it required considerable effort to optimize the parameters. Therefore, further study is required for the visu-

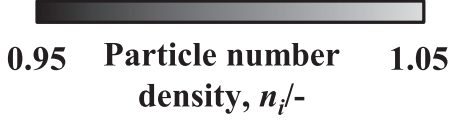

(a)

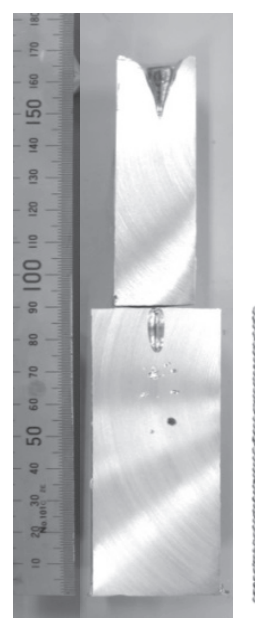

(b)

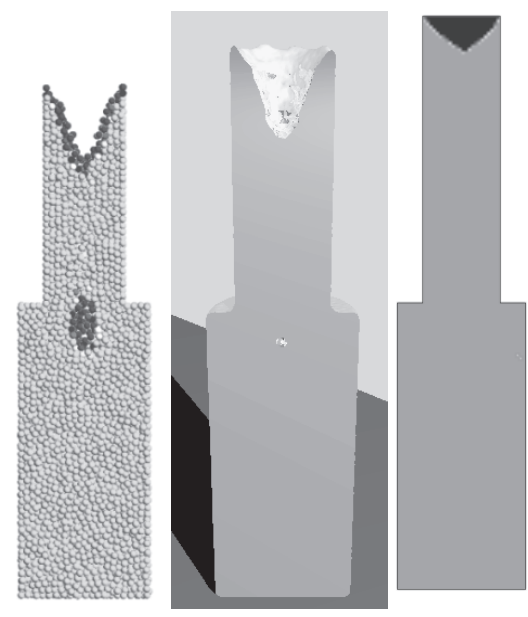

Fig. 7 Experimental and calculated results of shrinkage shape: $t_{\text {pour }}=14 \mathrm{~s}$. (a) Experimental result, (b) Particle number density, (c) Visualized by POV-RAY, (d) FDM. alization using the blob method with universality by such using the same weight function to the particle method calculation.

\subsubsection{Applicability to the shrinkage prediction}

Calculation elements of the particle method, called particles, are just representing points of continuum body. Therefore, the particle method is easily misunderstood by visualization using the spheroidal shape for each calculation element as if the elements are rigid and have a certain shape. In the MPS method used in this study, the particles are regarded as close-packed when the particles are arranged as a lattice structure. If the particles are visualized using spheroidal shaped objects, some empty space will be observed, misleading the existence of shrinkage porosity. In contrast, the attitude of the blob method is similar to that of the particle method in the existence of objects. Iso-surfaces of some scalar fields are visualized as surfaces in the blob method, whereas the iso-surfaces with low $n_{i}$ is determined as free surfaces in the particle method. Hirata et al. have succeeded to express complex shaped shrinkage by using the blob method ${ }^{19,21)}$. However, microscale porosities such as entrapped gas and porous shrinkage cavity are difficult to be directly expressed by using the blob method, because the sizes of the porosities are quite small compared to the particles. Therefore, both the visualization method and the evaluation method of various types of shrinkage porosities should be discussed to evaluate the defects in the castings.

Another issue is the case of alloys. Such as the solidification pattern, structure and rheological behavior should be considered to discuss the shrinkage formation. However, the proposed method based on the particle method enabled integrated simulation from the pouring to the end of solidification shrinkage formation directly, whereas the conventional methods usually calculate the flow and solidification shrinkage separately. The results show a high applicability of the particle method to perform further integrated simulations including deformation and segregation phenomena. On the other hands, there remains much to be solved in the particle method. The development of computers will help one of the most critical issues, the calculation speed. However, the other problem such as the oscillation of the velocity and pressure should be improved fundamentally.

\section{Conclusions}

Shrinkage formation behaviors under different pouring speeds were calculated using the integrated simulation of flow and solidification using the MPS method.

The improved multiple relaxation method significantly improved the velocity decay in the flow simulation, and also improve the stability of the calculation for the complex shaped castings.

Next, the flow simulation was combined with the solidification simulation, and shrinkage formation behaviors were calculated under different pouring speed. The proposed method enables to calculate shrinkage behavior and its influence on the flow simultaneously, and consequent shrinkage shape.

The results show a high applicability of the particle method to perform further integrated simulations. 


\section{REFERENCES}

1) I. Imafuku: Trans. Jpn. Soc. Mech. Eng. C. 47 (1981) 918-926.

2) I. Imafuku: Trans. Jpn. Soc. Mech. Eng. C. 48 (1982) 1959-1967.

3) I. Imafuku: Trans. Jpn. Soc. Mech. Eng. C. 48 (1982) 1968-1976.

4) I. Ohnaka, Y. Mori, Y. Nagasaka and T. Fukusako: IMONO 53 (1981) 673-679.

5) Y. Nagasaka, S. Kiguchi and M. Nachi: IMONO 61 (1989) 98-103.

6) I. Takahashi, T. Uchida and K. Anzai: J.JFS 78 (2006) 661.

7) J. Beech, M. Barkhudarov, K. Chang and S.B. Chin: Modeling of Casting Welding and Advanced Solidification Processes VIII (1998) 1071.

8) T. Kinoshita, S. Ueda and M. Matsushima: IMONO 37 (1965) 660.

9) M. Mohri: IMONO 61 (1989) 25.

10) S.Koshizuka: SuchiRyutaiRikigaku, (Baifukan, 1997).

11) S.Koshizuka: Ryushihou Simulation, (Baifukan, 2008).

12) S.Koshizuka: Ryushihou, (Maruzen, 2005).

13) M.Ichimiya and Y.Sakai: Reports of the 162th JFS Meeting, 162
(2013), 104.

14) M. Ichimiya and Y. Sakai: J.JFS 85 (2013) 481.

15) M.Kazama and T.Suwa: Reports of the 162th JFS Meeting, 162 (2013), 105.

16) P.W. Cleary: Appl. Math. Model. 34 (2010) 3189-3201.

17) N.Hirata and K.Anzai: Reports of the 152th JFS Meeting, 152 (2008), 34.

18) N.Hirata and K.Anzai: Reports of the 153th JFS Meeting, 153 (2008), 71.

19) N. Hirata and K. Anzai: Mater. Trans. 52 (2011) 1931-1938.

20) N. Hirata and K. Anzai: J.JFS 83 (2011) 259-267.

21) N. Hirata and K. Anzai: J.JFS 86 (2014) 127.

22) N. Hirata and K. Anzai: J.JFS 87 (2015) 702-708

23) N. Hirata and K. Anzai: J.JFS 80 (2008) 81-87.

24) The manual of POV-Ray 3.7 <http://www.povray.org/documentation/ 3.7.0/r3_4.html\#r3_4_5_1_1> 